\title{
CORRECTORS AND ERROR ESTIMATES IN THE HOMOGENIZATION OF A MULLINS-SEKERKA PROBLEM
}

\author{
Adriana GARRONI ${ }^{\mathrm{a}}$, Barbara NIETHAMMER ${ }^{\mathrm{b}}$

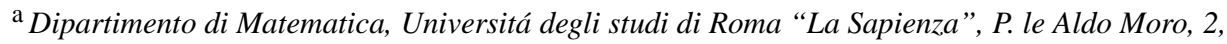 \\ 00185 Roma, Italy \\ ${ }^{\mathrm{b}}$ Inst. für Angew. Math., Universität Bonn, Wegelerstr. 6, 53115 Bonn, Germany
}

Received 15 January 2001, revised 3 March 2001

AbStRACT. - We study the homogenization of a Mullins-Sekerka free boundary problem which serves as a model for coarsening of nuclei in a first order phase transformation. We consider a regime where the volume fraction of the nuclei is small but screening effects are not negligible. The limit equation was recently derived in [12]. We improve this convergence result by constructing correctors and providing error estimates in terms of the volume fraction. This yields in particular an asymptotic expansion for the growth rate of the nuclei.

(C) 2002 L'Association Publications de l'Institut Henri Poincaré. Published by Elsevier B.V. All rights reserved

RÉSUMÉ. - On étudie l'homogénéisation d'un problème de frontiere libre de Mullins-Sekerka, qui sert de modèle pour le "coarsening" des noyaux dans une transformation de phase du premièr ordre. On considére un régime où la fraction de volume des noyaux est petite mais où les effets de criblage ne sont pas négligeables. L'équation limite a récemment été dérivée dans [12]. Nous améliorons ce résultat de convergence en construisant des correcteurs et fournissant des evaluations d'erreur en termes de la fraction de volume. Ceci conduit en particulier á une expansion asymptotique pour le taux de croissance des noyaux.

(C) 2002 L'Association Publications de l'Institut Henri Poincaré. Published by Elsevier B.V. All rights reserved

\section{Introduction}

The Mullins-Sekerka model describes the last stage of a first order phase transition. For example, consider an initially homogeneous two-component mixture which is quenched to sufficiently low temperatures such that the components want to separate. After an initial stage, two phases form. In each of the phases, the relative concentration has reached its respective bulk equilibrium value while the phases are separated by a thin

E-mail addresses: garroni@mercurio.mat.uniroma1.it (A. Garroni), Barbara.Niethammer@iam.uni-bonn.de (B. Niethammer). 
interfacial layer. Once, the sharp interface regime has emerged, the system is driven by reduction of surface energy and limited by diffusion. Since diffusion is fast compared to the velocity of the interface, one assumes that the diffusion field, here given by the chemical potential, is in quasi-steady equilibrium. This gives in dimensionless variables that the normal velocity $V$ of the interface is given by

$$
V=[\nabla \mu \cdot \vec{n}],
$$

where the chemical potential $\mu$ solves

$$
\begin{aligned}
-\Delta \mu=0 & \text { in each phase, } \\
\mu=\kappa & \text { on the interface. }
\end{aligned}
$$

Here $\vec{n}$ denotes the normal to the phase boundary, $[\nabla \mu \cdot \vec{n}]$ the jump of $\nabla \mu \cdot \vec{n}$ across the interface and $\kappa$ the mean curvature of the interface. Eq. (3) is the well-known GibbsThomson law for local equilibrium at the interface. The Mullins-Sekerka evolution preserves the volume fraction of the phases and decreases the surface area. Local existence results for smooth solutions were obtained in [4,6], global existence of weak solutions in [3].

In the following we are interested in the regime when the volume fraction of one phase is very small. Then this phase consists of many disconnected components, in the following called nuclei or particles, which quickly become radially symmetric and do essentially not move in space. In order to reduce the surface energy, large particles grow at the expense of small particles which eventually vanish - a phenomenon known as Ostwald ripening. We use the fact that particles are almost spherical to simplify the model by averaging (1) for each particle $P_{i}$ such that

$$
V_{P_{i}}:=f_{P_{i}}[\nabla \mu \cdot \vec{n}] .
$$

Then each particle is represented by its radius $R_{i}(t)$, the curvature is given by $1 / R_{i}$ and the normal velocity by $\dot{R}_{i}(t)$. Formal asymptotics in [16] and a stability analysis in $[1,2]$ justify this simplification in the regime of small volume fraction. Note that the chemical potential inside the balls is now given by $\mu=1 / R_{i}$ and (4) becomes

$$
V_{P_{i}}:=f_{P_{i}} \nabla \mu \cdot \vec{n},
$$

where $\vec{n}$ denotes the outer normal to the particle and $\nabla \mu \cdot \vec{n}$ is taken from outside the particle. Since the system is now completely determined by the radii $\left\{R_{i}\right\}$ one would like to derive an evolution law for $R_{i}$ at least in the regime of a large number $n$ of particles with very small volume fraction $\varphi$, i.e., in the sense of homogenization. For that, one has to understand the range of particle interactions which are limited by screening: one particle is screened from particles far away by particles in the neighbourhood. It is found (cf. [12]) that screening effects are relevant if $n \approx 1 / \varphi^{1 / 2}$, which is equivalent to the particles having capacity density of order 1 . In this regime the homogenization limit as 
$\varphi \rightarrow 0$ was identified in [12]. One introduces the particle size distribution $v$ which is defined such that $\int_{D} \int_{0}^{r} v(t, \mathrm{~d} x, \mathrm{~d} \tilde{r})$ gives the fraction of existing particles at time $t$ with center in $D$ and radius smaller than $r$. It is shown in [12] that in the homogenization limit the particle size distribution solves

$$
\partial_{t} v+\partial_{r}\left(\frac{1}{r^{2}}(r u(t, x)-1) v\right)=0
$$

where the limit of the chemical potential - now denoted by $u$ - satifies

$$
-\Delta u+4 \pi\left(u \int r v \mathrm{~d} r-\int v \mathrm{~d} r\right)=0 .
$$

This system is an extension of the classical theory for Ostwald Ripening by Lifshitz, Slyozov and Wagner ("LSW") [10,18] where space dependence is neglected and $u$ is determined by conservation of total volume fraction. The LSW model is derived rigorously in [11] by homogenization in the regime $n \ll 1 / \varphi^{1 / 2}$ which leads to the mathematical setting of particles with vanishing capacity density.

While the work [12] identifies the limit equation, it does not provide any further qualitative description of the solution. Thus, it is the aim of this paper to find under the same assumptions on the system as in [12] correctors and provide error-estimates. This will also put future numerical experiments on a more solid basis.

The outline of the paper is as follows. We indicate the scaling in Section 2.1, introduce some notation in Section 2.2 and present the precise setting for our analysis in Section 2.3. The homogenization limit was identifed in [12] and we summarize the main results in Theorem 2.1 in Section 2.4. Guided by the form of the limit equation for the chemical potential we construct a corrector in Section 3. In Section 4 (cf. Theorem 4.1) we prove an error estimate for the difference between the chemical potential and the corrector. The proof extends ideas of $[5,8]$ to our time dependent, nonperiodic setting. Finally, in Section 5 we prove an asymptotic expansion for the evolution of the radii (Theorem 5.1). The proof relies on the corrector result Theorem 4.1 and a local uniform estimate away from the particles which is given in Lemma 5.2.

Finally, let us briely mention another issue in the LSW-theory. By an asymptotic analysis LSW predict that all solutions exhibit a universal long-time behavior. However, the mathematical paper [13] shows, that, contrary to the predictions, the large time asymptotics depend sensitively on the data, more precisely on the behavior of the initial distribution at the end of the support. Naturally, one might ask, what the selection criterion for the long-time asymptotics of (5), (6) is. However, it seems quite difficult to provide a rigorous mathematical analysis and we plan to investigate this issue by careful simulations.

For more information on the theory of Ostwald Ripening and the physical background we refer to [12] and the references therein. 


\section{The setting and preliminaries}

\subsection{The scaling}

In this section we recall the appropriate scaling, which is derived in [12, Ch. 3]. We consider a finite or periodic box in $\mathbb{R}^{3}$ of length $\mathrm{L}$ filled with $n$ particles with mean radius $\mathcal{R}$ which have small volume fraction $\varphi$. The average distance $d$ is given by $d=\mathcal{R} / \varphi^{1 / 3}$ and it is found by formal considerations (cf. [9,12]) that the range of particle interactions, the screening length $\xi$, is given by

$$
\xi=\frac{\mathcal{R}}{\varphi^{1 / 2}} .
$$

In the regime $\varphi \ll 1$ we find $\xi \gg d \gg \mathcal{R}$, hence one has a nice separation of length scales. It is natural to rescale the space with respect to $\xi$ and we assume in the following that the size of the box is of the order of the screening length, for simplicity $L=\xi$. We introduce the rescaled variables $\hat{x}, \hat{t}, \widehat{R}_{i}$ and $u$ via

$$
\begin{aligned}
x & =\xi \hat{x}, \\
t & =\mathcal{R}^{3} \hat{t}, \\
R_{i} & =\mathcal{R} \widehat{R}_{i}, \\
\mu(t, x) & =\frac{1}{\mathcal{R}} u(\hat{t}, \hat{x}) .
\end{aligned}
$$

The scaling of the chemical potential is motivated by the Gibbs-Thomson condition on the phase boundary, whereas the time scale is such that the limit evolution is nontrivial.

\subsection{Notation}

From now on we drop the hats and introduce for a simpler notation the parameter $\varepsilon:=\varphi^{1 / 6}$. The scaling in Section 2.1 implies that we work in the unit cube $\Omega:=(0,1)^{3}$, that the particles have mean distance $\varepsilon$ and the number of particles is bounded by $1 / \varepsilon^{3}$. The particle centers will be denoted by $X_{i}$ and the particles themselves by

$$
B_{i}(t):=B\left(X_{i}, \varepsilon^{3} R_{i}(t)\right) \quad \text { with } R_{i}(t) \in[0, \infty) .
$$

Note, that $X_{i}, R_{i}$ and $B_{i}$ depend on $\varepsilon$, but for an easier reading we will in the following neglect this, since the index $i$ already indicates the dependence on $\varepsilon$. We have to assume that particle centers $X_{i}$ are well separated in the sense that there exists a $\lambda>0$ such that

$$
\left\{B\left(X_{i}, \lambda \varepsilon\right)\right\}_{i} \text { are disjoint for all } \varepsilon>0 .
$$

We denote for any $t \in[0, \infty)$

$$
\Omega^{\varepsilon}(t):=\Omega \backslash \bigcup_{i: R_{i}(t)>0} \overline{B_{i}(t)}
$$


Furthermore we define the joint distribution $v_{t}^{\varepsilon}$ of particle centers and radii at a given time $t$ by

$$
\int \zeta \mathrm{d} v_{t}^{\varepsilon}=\varepsilon^{3} \sum_{i} \zeta\left(X_{i}, R_{i}(t)\right) \quad \text { for } \zeta \in C_{\#}^{0},
$$

where $\int:=\int_{\Omega \times(0, \infty)}$ and

$C_{\#}^{0}:=\left\{\zeta=\zeta(x, r) \mid \zeta \in C^{0}\left(\mathbb{R}^{3} \times(0, \infty)\right), \zeta(\cdot, r)\right.$ is $\Omega$-periodic, $\left.\zeta(x, \cdot) \in C_{0}^{0}((0, \infty))\right\}$.

Note that since $\zeta(x, 0)=0$ for all $\zeta \in C_{\#}^{0}$ the sum in (8) effectively only extends over the 'active' particles, i.e., the $i$ such that $R_{i}(t)>0$. In the following we will always use the notation

$$
\sum_{i}:=\sum_{i: X_{i} \in \Omega, R_{i}(t)>0} .
$$

Trivially, with this notation we have for all times $t$ that

$$
\int \mathrm{d} \nu_{t}^{\varepsilon}=\varepsilon^{3} \sum_{i} 1 \leqslant 1
$$

Our scaling is also such that for the initial radii $R_{i 0}$

$$
\varepsilon^{3} \sum_{i} R_{i 0}^{3}=\int r^{3} \mathrm{~d} \nu_{0}^{\varepsilon}=1
$$

In addition the appropriate space for the chemical potential will be

$$
H_{\#}^{1}(\Omega):=\left\{u \in H_{\mathrm{loc}}^{1}\left(\mathbb{R}^{3}\right) \mid u \text { is } \Omega \text {-periodic }\right\} .
$$

\subsection{The problem}

With the scaling introduced in Section 2.1 and the notation from Section 2.2 our precise mathematical problem takes the following form. We are looking for a solution

$$
\begin{aligned}
& \dot{R}_{i}(t)=\frac{1}{4 \pi \varepsilon^{3} R_{i}{ }^{2}} \int_{\partial B_{i}} \nabla u^{\varepsilon} \cdot \vec{n} \quad \text { as long as } R_{i}(t)>0, \\
& R_{i}(0)=R_{i 0},
\end{aligned}
$$

where $u^{\varepsilon}(t, \cdot) \in H_{\#}^{1}(\Omega)$ is determined via

$$
\begin{aligned}
-\Delta u^{\varepsilon} & =0 & & \text { in } \Omega^{\varepsilon}(t), \\
u^{\varepsilon} & =\frac{1}{R_{i}(t)} & & \text { in } B_{i} \text { if } R_{i}(t)>0 .
\end{aligned}
$$




\subsection{Well-posedness, a priori estimates and the limit equation}

It is not difficult to prove existence of a smooth solution locally in time for (11), (12) (cf. [11] and the references therein) and one can extend the solution up to a time where either two balls touch or some balls shrink to a point. Our evolution conserves the volume fraction of particles, i.e.,

$$
\varepsilon^{3} \sum_{i} R_{i}^{3}(t)=\int r^{3} \mathrm{~d} v_{t}^{\varepsilon}=\int r^{3} \mathrm{~d} v_{0}^{\varepsilon} \stackrel{(10)}{=} 1
$$

for all $t>0$, which follows by integrating (12) and using (11). Note that from (9) and (13) it follows by Hölder's inequality that

$$
\varepsilon^{3} \sum_{i} R_{i}^{\alpha}(t)=\int r^{\alpha} \mathrm{d} v_{t}^{\varepsilon} \leqslant 1 \quad \text { for all } \alpha \in[0,3] .
$$

Furthermore the surface area decreases, i.e., for all $t \geqslant 0$ it holds

$$
\int r^{2} \mathrm{~d} v_{t}^{\varepsilon}+\frac{1}{2 \pi} \int_{0}^{t} \int_{\Omega}\left|\nabla u^{\varepsilon}\right|^{2}=\int r^{2} \mathrm{~d} v_{0}^{\varepsilon} \leqslant 1 .
$$

To see (15) one multiplies (12) with $u^{\varepsilon}$, integrates by parts and uses the boundary conditions for $\dot{R}_{i}$ and $u^{\varepsilon}$. Return to the problem that two balls might touch. Volume conservation (13) implies that

$$
\sup _{i} R_{i}(t) \leqslant \frac{1}{\varepsilon} .
$$

Hence, the maximal radius of a ball is $\varepsilon^{2}$ which is smaller than $\lambda \varepsilon$ for sufficiently small $\varepsilon>0$ and thus balls cannot touch. The second possibility, that existence of a smooth solution breaks down, occurs at times when a particle shrinks to a point at time $t_{1}$. Then we update the initial data and start again with data $R_{i}\left(t_{1}\right)$. Proceeding in this way we obtain a continuous piecewise smooth solution $R_{i}$ of (11).

In the following we investigate the limit $\varepsilon \rightarrow 0$. To ensure that the scaling remains the right one, we have to make the additional assumption that initially not too much of the volume is taken by few very large particles, i.e., we assume that

$$
\int_{r>M} r^{3} \mathrm{~d} \nu_{0}^{\varepsilon} \rightarrow 0 \quad \text { as } M \rightarrow \infty \text { uniformly in } \varepsilon .
$$

In a slightly different setting the limit for $\varepsilon \rightarrow 0$ is identified in [12]. We state a theorem below which is adapted to our setting and can be proved in exactly the same way as Theorem 3.1 in [12].

THEOREM 2.1 ([12], Theorem 3.1). - There exists a subsequence, again denoted by $\varepsilon \rightarrow 0$, such that 


$$
\begin{array}{rlrl}
\int \zeta \mathrm{d} v_{t}^{\varepsilon} & \rightarrow \int \zeta \mathrm{d} v_{t} & & \text { locally uniformly in } t \in[0, \infty) \text { for all } \zeta \in C_{\#}^{0}, \\
u^{\varepsilon} & \rightarrow u & \text { weakly in } L_{\mathrm{loc}}^{2}\left([0, \infty) ; H_{\#}^{1}(\Omega)\right) .
\end{array}
$$

The limits $v_{t}$ and $u$ are weak solutions of Eqs. (5) and (6) respectively, i.e.,

$$
\frac{\mathrm{d}}{\mathrm{d} t} \int \zeta \mathrm{d} v_{t}=\int \partial_{r} \zeta \frac{1}{r^{2}}(r u(t)-1) \mathrm{d} v_{t}
$$

holds in $\mathcal{D}^{\prime}((0, \infty))$ for all $\zeta \in C_{\#}^{0}$ with $\partial_{r} \zeta \in C_{\#}^{0}$, and

$$
\int_{\Omega} \nabla u(t) \cdot \nabla \varphi \mathrm{d} x+4 \pi \int \varphi u(t) r \mathrm{~d} v_{t}=4 \pi \int \varphi \mathrm{d} v_{t}
$$

for all $\varphi=\varphi(x) \in H_{\#}^{1}(\Omega)$ and a.e. $t \in(0, \infty)$.

We remark that we need (17) to obtain a nontrivial limit; indeed it holds

$$
\int r^{3} \mathrm{~d} v_{t}=\int r^{3} \mathrm{~d} v_{0}=1 \quad \text { for all } t \in[0, \infty) .
$$

Furthermore, by assumption (7) one can show that the marginal of $v_{t}$ w.r.t. $x$ has a Lebesgue density, that is there exists a constant $C$ such that

$$
\int \varphi \mathrm{d} v_{t} \leqslant C \int_{\Omega} \varphi \mathrm{d} x \quad \text { for all integrable } \varphi=\varphi(x) \text { and } t \in[0, \infty) \text {. }
$$

\section{Construction of a corrector}

The procedure in [12] identifies the limit equation of the homogenization of (11), (12), but does not give any qualitative estimates. It is the aim of this paper to provide a better qualitative description of the functions $R_{i}$ and $u^{\varepsilon}$ in form of corrector estimates for $u^{\varepsilon}$ from which an asymptotic expansion for $R_{i}$ follows.

To simplify the presentation we will in the following assume that the particle centers $X_{i}$ sit on a lattice of spacing $\varepsilon$ such that (7) holds with $\lambda=1 / 2$. Furthermore we will not explicitly state in each single statement that the assertion holds for sufficiently small $\varepsilon$ and that the constants depend on $\Omega$. In addition, we will always consider a sequence $\varepsilon \rightarrow 0$ such that the convergence stated in Theorem 2.1 holds.

First we have to introduce some auxiliary functions. We denote by $Q_{i}:=\left(X_{i}-\frac{\varepsilon}{2}\right.$, $\left.X_{i}+\frac{\varepsilon}{2}\right)^{3}$ the cube with center $X_{i}$ and side length $\varepsilon$. The characteristic function of $Q_{i}$ is denoted by $\chi_{Q_{i}}$ and we introduce the step functions

$$
R^{\varepsilon}(t, x):=\sum_{i} \chi_{Q_{i}}(x) R_{i}(t) \quad \text { and } \quad \chi^{\varepsilon}(t, x):=\sum_{i} \chi_{Q_{i}}(x) .
$$

Note that $R^{\varepsilon}(\cdot, x)$ is continuous for all $x \in \Omega$ and by (13) it is uniformly bounded in $L^{\infty}\left((0, \infty) ; L^{3}(\Omega)\right)$. The function $\chi^{\varepsilon}$ is just the characteristic function of the union of 
all cubes which contain a particle of positive volume. $\chi^{\varepsilon}(\cdot, x)$ is right continuous for all $x \in \Omega$ and $0 \leqslant \chi^{\varepsilon} \leqslant 1$.

Denote by $\bar{R}$ and $\bar{\chi}$ the weak limits of $R^{\varepsilon}$ and $\chi^{\varepsilon}$. Then it follows with the notation in Section 2 from (9), (13) and (18) that for all continuous, $\Omega$-periodic functions $\varphi=\varphi(x)$

$$
\int \varphi R^{\varepsilon} \mathrm{d} x \rightarrow \int \varphi r \mathrm{~d} v_{t}=\int \varphi \bar{R}(t, x) \mathrm{d} x
$$

and

$$
\int \varphi \chi^{\varepsilon} \mathrm{d} x \rightarrow \int \varphi \mathrm{d} v_{t}=\int \varphi \bar{\chi}(t, x) \mathrm{d} x
$$

locally uniformly in $t \in[0, \infty)$. With this notation we can rewrite (6) as

$$
-\Delta u+4 \pi(\bar{R} u-\bar{\chi})=0 \quad \text { in } \Omega .
$$

Now we introduce an appropriate approximation of $u$ on the $\varepsilon$-level. For each $t$ we denote by $z^{\varepsilon}(t, \cdot)$ the solution of the following elliptic problem

$$
-\Delta z^{\varepsilon}+4 \pi\left(R^{\varepsilon} z^{\varepsilon}-\chi^{\varepsilon}\right)=0 \quad \text { in } \Omega, \quad z^{\varepsilon}(t, \cdot) \in H_{\#}^{1}(\Omega) .
$$

With (23) we see - at least formally - that $z^{\varepsilon}$ should be a good approximation of $u$. Indeed, we will prove in Lemma 3.3 that for almost all times $z^{\varepsilon}$ converges strongly to $u$ in $W^{1, p}(\Omega)$ for all $p<\infty$. The following lemma gives a general existence result for the type of elliptic equation (23) and (24).

LEMma 3.1. - Consider the equation

$$
-\Delta u+g u=f \quad \text { in } \Omega, \quad u \in H_{\#}^{1}(\Omega),
$$

where $g, f \in L^{3}(\Omega),\|g\|_{L^{3}(\Omega)}+\|f\|_{L^{3}(\Omega)} \leqslant C_{1}$ and $g \geqslant 0$ such that $\int_{\Omega} g \geqslant c_{2}>0$.

There exists a unique solution $u \in H_{\#}^{1}(\Omega) \cap W^{2,3}(\Omega)$ such that

$$
\|u\|_{W^{2,3}(\Omega)} \leqslant C\left(C_{1}, c_{2}\right) .
$$

Proof. - Arguing by contradiction one finds that there exists a constant $c=c\left(c_{2}, C_{1}\right)$ such that

$$
c\|u\|_{H^{1}(\Omega)}^{2} \leqslant \int_{\Omega}|\nabla u|^{2}+g|u|^{2} \mathrm{~d} x
$$

holds for all $u \in H^{1}(\Omega)$. Then the lemma follows by a standard application of the LaxMilgram lemma and regularity results for elliptic equations (see, e.g., [7, Ch. 4]).

COROLlaRY 3.2. - For any $t \in(0, \infty)$ there exists a unique solution $z^{\varepsilon}(t, \cdot) \in$ $H_{\#}^{1}(\Omega) \cap W^{2,3}(\Omega)$ to $(24)$ such that for any $T<\infty$

$$
\sup _{t \in(0, T)}\left\|z^{\varepsilon}(t, \cdot)\right\|_{W^{2,3}(\Omega)} \leqslant C(T) .
$$


This implies, with the embedding theorem from Sobolev into Hölder spaces, that $z^{\varepsilon}(t, \cdot)$ is Hölder-continuous for any $\alpha<1$ and that $z^{\varepsilon}(t, \cdot) \in W^{1, p}(\Omega)$ for all $p<\infty$ with

$$
\sup _{t \in(0, T)}\left\|z^{\varepsilon}(t, \cdot)\right\|_{C^{0, \alpha}(\Omega)}+\sup _{t \in(0, T)}\left\|z^{\varepsilon}(t, \cdot)\right\|_{W^{1, p}(\Omega)} \leqslant C(\alpha, p, T) .
$$

Proof. - We know from (21) that

$$
\int_{\Omega} R^{\varepsilon}(t, x) \mathrm{d} x \rightarrow \int r \mathrm{~d} v_{t}
$$

locally uniformly in $t \in[0, \infty)$. Since $\int r^{3} \mathrm{~d} v_{t}=1$ for all $t \geqslant 0$, it must hold that $\int r \mathrm{~d} v_{t}>0$ for all $t>0$. Hence, we conclude that for all $T<\infty$ there exists a constant $c_{T}>0$ such that for sufficiently small $\varepsilon$

$$
\int_{\Omega} R^{\varepsilon}(t, x) \mathrm{d} x \geqslant c_{T}>0 .
$$

Since $0 \leqslant \chi^{\varepsilon} \leqslant 1$ and $R^{\varepsilon}$ is uniformly bounded in $L^{\infty}\left((0, \infty) ; L^{3}(\Omega)\right)$ by (13) the result follows from Lemma 3.1.

LEMMA 3.3. - For almost all $t \in(0, \infty)$ it holds

$$
z^{\varepsilon}(t, \cdot) \rightarrow u(t, \cdot) \quad \text { in } W^{1, p}(\Omega) \text { for all } p<\infty
$$

Proof. - We take $z^{\varepsilon}-u$ as a test function in the difference of (24) and (23). We fix $T<\infty$ and use (26) to obtain for almost all $t \in(0, T)$

$$
\begin{aligned}
c_{T}\left\|z^{\varepsilon}(t, \cdot)-u(t, \cdot)\right\|_{H^{1}(\Omega)}^{2} \leqslant & 4 \pi\left|\int_{\Omega}\left(\chi^{\varepsilon}-\bar{\chi}\right)\left(z^{\varepsilon}-u\right)(t, \cdot) \mathrm{d} x\right| \\
& +4 \pi\left|\int_{\Omega} z^{\varepsilon}\left(R^{\varepsilon}-\bar{R}\right)\left(z^{\varepsilon}-u\right)(t, \cdot) \mathrm{d} x\right| .
\end{aligned}
$$

We find with Hölder's inequality, (27) and the properties of $\chi^{\varepsilon}$ and $R^{\varepsilon}$ that

$$
\sup _{t \in(0, T)}\left\|z^{\varepsilon}(t, \cdot)-u(t, \cdot)\right\|_{H^{1}(\Omega)}^{2} \leqslant C_{T}
$$

Now we fix $t \in(0, T)$ such that $u(t, \cdot)$ is defined. There exists a subsequence $\varepsilon=\varepsilon(t)$ such that $z^{\varepsilon}(t, \cdot)-u(t, \cdot)$ converges strongly in $L^{2}(\Omega)$ to some limit. It follows from (21), (22) and (29) that for this subsequence

$$
\left\|z^{\varepsilon}(t, \cdot)-u(t, \cdot)\right\|_{H^{1}(\Omega)}^{2} \rightarrow 0
$$

Since $u(t, \cdot)$ is uniquely determined by Lemma 3.1 we conclude that (30) holds for the whole sequence. With Corollary 3.2 and the Sobolev embedding we find (28). 
With the help of $z^{\varepsilon}$ we are going to construct a corrector for $u^{\varepsilon}$. For that we use similarly as in $[5,8]$ the capacity potential of $B_{i}$ in

$$
T_{i}:=B\left(X_{i}, \frac{\varepsilon}{2}\right) .
$$

This is given as the solution of the equation

$$
\begin{aligned}
-\Delta w_{i}=0 & \text { in } T_{i} \backslash \overline{B_{i}}, \\
w_{i}=0 & \text { in } B_{i}, \\
w_{i}=1 & \text { on } \partial T_{i} .
\end{aligned}
$$

To be precise, with this definition $1-w_{i}$ is the capacity potential of $B_{i}$ with respect to $T_{i}$. One easily computes that

$$
w_{i}(t, x)=\frac{\frac{1}{\left|x-X_{i}\right|}-\frac{1}{\varepsilon^{3} R_{i}}}{\frac{2}{\varepsilon}-\frac{1}{\varepsilon^{3} R_{i}}}=\frac{1}{1-2 \varepsilon^{2} R_{i}}\left(1-\frac{\varepsilon^{3} R_{i}}{\left|x-X_{i}\right|}\right) \quad \text { in } T_{i} \backslash \overline{B_{i}} .
$$

We extend $w_{i}$ by 1 to $\Omega \backslash \bigcup_{i} T_{i}$ and by 0 to $B_{i}$ and define

$$
w^{\varepsilon}(t, x):=\sum_{i} \chi_{Q_{i}}(x) w_{i}(t, x)
$$

and

$$
g^{\varepsilon}(t, x):=\sum_{i} \chi_{Q_{i}}(x) \frac{1-w_{i}(t, x)}{R_{i}(t)} .
$$

Notice that $g^{\varepsilon}$ is harmonic in $T_{i} \backslash \overline{B_{i}}, g^{\varepsilon}=1 / R_{i}$ on $\partial B_{i}$ and $g^{\varepsilon}=0$ on $\partial T_{i}$. Our candidate for a corrector is

$$
v^{\varepsilon}:=z^{\varepsilon} w^{\varepsilon}+g^{\varepsilon} .
$$

In the rest of this paper we are going to show the following error estimates (cf. Theorems 4.1 and 5.1).

$$
\sup _{t \in(0, T)}\left\|\nabla\left(u^{\varepsilon}-v^{\varepsilon}\right)(t, \cdot)\right\|_{L^{2}(\Omega)} \leqslant C(T) \varepsilon
$$

and

$$
\sup _{t \in(0, T)}\left|\dot{R}_{i} R_{i}^{2}-\left(R_{i} z^{\varepsilon}\left(X_{i}\right)-1\right)\right| \leqslant C(T) R_{i} \varepsilon^{1 / 2} .
$$

A future aim will be to transform these results into error estimates for the particle size distribution. It seems natural that for this aim one should use a corrector of the form $u w^{\varepsilon}+g^{\varepsilon}$. Indeed we find that $u^{\varepsilon}-\left(u w^{\varepsilon}+g^{\varepsilon}\right)$ converges strongly to zero in $H^{1}(\Omega)$ (cf. Corollary 4.2). However, to obtain an error estimate like in (36), we have to start from an error estimate for the data, and carry this over to the solution at a given time. This 
seems feasible if one assumes strong convergence of $R^{\varepsilon}(0, \cdot)$, an assumption which is not satisfying from the point of view of applications. The crucial point will be to find the right metric to deal with the weak convergences. So far a related problem has been only attacked for the limit problem in the space independent case in [14]. There wellposedness is shown with respect to the $L^{\infty}$-Wasserstein distance for the particle size distributions.

\section{An error estimate for $u^{\varepsilon}-v^{\varepsilon}$}

The aim of this section is to prove an error estimate for $u^{\varepsilon}-v^{\varepsilon}$ in the spirit of [8], which treats a time-independent setting where all particles are equal.

THEOREM 4.1. - Let $u^{\varepsilon}$ be the solution to (12) and $v^{\varepsilon}$ be defined as in (35). For any $T<\infty$ we have

$$
\sup _{t \in(0, T)}\left\|\nabla\left(u^{\varepsilon}-v^{\varepsilon}\right)(t, \cdot)\right\|_{L^{2}(\Omega)} \leqslant C(T) \varepsilon .
$$

Proof. - We first collect several facts which we will need in the forthcoming proof.

- Poincaré inequality on $Q_{i}$. For any $y^{\varepsilon} \in H^{1}\left(Q_{i}\right)$ such that $y^{\varepsilon}=0$ in $B_{i}$ it holds

$$
\left\|y^{\varepsilon}\right\|_{L^{2}\left(Q_{i}\right)} \leqslant \frac{C}{R_{i}^{1 / 2}}\left\|\nabla y^{\varepsilon}\right\|_{L^{2}\left(Q_{i}\right)} .
$$

The inequality follows, e.g., from [19, Corollary 4.5.2] and scaling, but one can also easily verify it by hand.

In addition we will need a Poincaré inequality on the whole domain:

- Poincaré inequality on $\Omega$. We show that for any $t \in(0, T)$ and any $y^{\varepsilon} \in H^{1}(\Omega)$ with $y^{\varepsilon}=0$ in $\bigcup_{i} B_{i}$ it holds

$$
\left\|y^{\varepsilon}\right\|_{L^{2}(\Omega)} \leqslant C(T)\left\|\nabla y^{\varepsilon}\right\|_{L^{2}(\Omega)} .
$$

For that we will show that

$$
\left\|y^{\varepsilon}\right\|_{L^{2}(\Omega)}^{2} \leqslant \frac{C}{\left(\sum_{i} \varepsilon^{3}\left(R_{i}(t)\right)^{1 / 2}\right)^{2}}\left\|\nabla y^{\varepsilon}\right\|_{L^{2}(\Omega)}^{2} .
$$

Similary as in the proof of Corollary 3.2 we conclude that

$$
\sup _{t \in(0, T)} \frac{1}{\sum_{i} \varepsilon^{3}\left(R_{i}(t)\right)^{1 / 2}} \leqslant C_{T}
$$

and (40) follows. To prove (41) we use again Corollary 4.5.2 from [19] which gives for $y \in H^{1}(\Omega), y=0$ in $\bigcup_{i} B_{i}$, that

$$
\int_{\Omega}|y|^{2} \leqslant \frac{C}{\operatorname{cap}(\{u=0\} ; \Omega)} \int_{\Omega}|\nabla y|^{2} \leqslant \frac{C}{\operatorname{cap}\left(\bigcup B_{i} ; \Omega\right)} \int_{\Omega}|\nabla y|^{2} .
$$


Thus, we have to show that

$$
\operatorname{cap}\left(\bigcup B_{i} ; \Omega\right) \geqslant c\left(\sum_{i} \varepsilon^{3} R_{i}^{1 / 2}\right)^{2} .
$$

Let $\varphi^{\varepsilon}$ be the capacity potential of $\bigcup_{i} B_{i}$ in $\Omega$, that is,

$$
\begin{aligned}
-\Delta \varphi^{\varepsilon}=0 & \text { in } \Omega \backslash \bigcup_{i} B_{i}, \\
\varphi^{\varepsilon}=1 & \text { in } B_{i}, \\
\varphi^{\varepsilon}=0 & \text { on } \partial \Omega .
\end{aligned}
$$

By (39) we have for all $i$ such that $R_{i}>0$ that

$$
\left\|\varphi^{\varepsilon}-1\right\|_{L^{2}\left(Q_{i}\right)}^{2} \leqslant \frac{C}{R_{i}}\left\|\nabla \varphi^{\varepsilon}\right\|_{L^{2}\left(Q_{i}\right)}^{2} .
$$

With

$$
S:=\left\{i: R_{i}>0 \text { and }\left\|\varphi^{\varepsilon}-1\right\|_{L^{2}\left(Q_{i}\right)}^{2} \geqslant \frac{1}{4} \varepsilon^{3}\right\}
$$

we obtain by summing over $i \in S$ that

$$
\sum_{i \in S} \varepsilon^{3} R_{i} \leqslant C \sum_{i}\left\|\nabla \varphi^{\varepsilon}\right\|_{L^{2}\left(Q_{i}\right)}^{2} \leqslant C\left\|\nabla \varphi^{\varepsilon}\right\|_{L^{2}(\Omega)}^{2} .
$$

If $i \notin S$ we have $\left\|\varphi^{\varepsilon}\right\|_{L^{2}\left(Q_{i}\right)}^{2} \geqslant \frac{1}{4} \varepsilon^{3}$ and thus, by the ordinary Poincaré inequality,

$$
\begin{aligned}
\left(\sum_{i \notin S} \varepsilon^{3} R_{i}^{1 / 2}\right)^{2} & \leqslant C\left(\sum_{i \notin S} \varepsilon^{3 / 2} R_{i}^{1 / 2}\left\|\varphi^{\varepsilon}\right\|_{L^{2}\left(Q_{i}\right)}\right)^{2} \\
& \leqslant C\left(\sum_{i \notin S} \varepsilon^{3} R_{i}\right)\left(\sum_{i}\left\|\varphi^{\varepsilon}\right\|_{L^{2}\left(Q_{i}\right)}^{2}\right) \\
& \stackrel{(14)}{\leqslant} C\left\|\nabla \varphi^{\varepsilon}\right\|_{L^{2}(\Omega)}^{2} .
\end{aligned}
$$

We notice that $\left(\sum_{i} \varepsilon^{3} R_{i}^{1 / 2}\right)^{2} \leqslant \sum_{i} \varepsilon^{3} R_{i}$ which yields with (44) and (45) the desired inequality (43).

- For any $q^{\varepsilon} \in H^{1}\left(Q_{i}\right)$ with $\int_{Q_{i}} q^{\varepsilon}=0$ it holds

$$
\int_{\partial T_{i}}\left|q^{\varepsilon}\right| \leqslant C \varepsilon^{3 / 2}\left(\int_{Q_{i}}\left|\nabla q^{\varepsilon}\right|^{2}\right)^{1 / 2}
$$

with $T_{i}=B\left(X_{i}, \varepsilon / 2\right)$. This follows by the Poincaré inequality for functions with mean value zero, the embedding $H^{1}\left(Q_{i}\right)$ into $L^{1}\left(\partial T_{i}\right)$ and a simple scaling argument. 
- Properties of $w_{i}$ and $w^{\varepsilon}$. The functions $w_{i}$ and $w^{\varepsilon}$ as defined in (32) and (33) respectively satisfy

$$
\begin{aligned}
\left\|1-w_{i}\right\|_{L^{p}\left(Q_{i}\right)} & \leqslant \frac{C R_{i}}{(3-p)^{1 / p}} \varepsilon^{2+3 / p} \quad \text { for } p \in[1,3), \\
\left\|1-w^{\varepsilon}(t, \cdot)\right\|_{L^{p}(\Omega)} & \leqslant \frac{C}{(3-p)^{1 / p}} \varepsilon^{2} \quad \text { for } p \in[1,3), \\
\left\|\nabla w_{i}\right\|_{L^{2}\left(Q_{i}\right)} & \leqslant C\left(R_{i} \varepsilon^{3}\right)^{1 / 2}, \\
\left\|\nabla w^{\varepsilon}(t, \cdot)\right\|_{L^{2}(\Omega)} & \leqslant C, \\
\nabla w_{i} \cdot \vec{n} & =\frac{4 \varepsilon R_{i}}{1-2 \varepsilon^{2} R_{i}} \quad \text { on } \partial T_{i}, \\
\frac{1}{\varepsilon^{3}} \int_{\partial T_{i}} \nabla w_{i} \cdot \vec{n} & =\frac{4 \pi R_{i}}{1-2 \varepsilon^{2} R_{i}} .
\end{aligned}
$$

One notes that

$$
1-\frac{1}{1-2 \varepsilon^{2} R_{i}} \leqslant 4 \varepsilon^{2} R_{i} \stackrel{(16)}{\leqslant} 4 \varepsilon
$$

and the rest follows easily by direct computation using (14).

We commence the proof of Theorem 4.1. We first observe that

$$
-\Delta\left(u^{\varepsilon}-v^{\varepsilon}\right)=-\Delta u^{\varepsilon}+\operatorname{div}\left(w^{\varepsilon} \nabla z^{\varepsilon}+z^{\varepsilon} \nabla w^{\varepsilon}+\nabla g^{\varepsilon}\right) \quad \text { in } \Omega^{\varepsilon}(t) .
$$

We test this equation with $u^{\varepsilon}-v^{\varepsilon}$ and by the fact that $u^{\varepsilon}-v^{\varepsilon}=0$ in $B_{i}$ we find

$$
\begin{aligned}
\int_{\Omega}\left|\nabla\left(u^{\varepsilon}-v^{\varepsilon}\right)\right|^{2}= & -\int_{\Omega} w^{\varepsilon} \nabla z^{\varepsilon} \cdot \nabla\left(u^{\varepsilon}-v^{\varepsilon}\right)+\int_{\Omega} \nabla z^{\varepsilon} \cdot \nabla w^{\varepsilon}\left(u^{\varepsilon}-v^{\varepsilon}\right) \\
& -\sum_{i}\left\{\int_{\partial T_{i}} z^{\varepsilon}\left(u^{\varepsilon}-v^{\varepsilon}\right) \nabla w_{i} \cdot \vec{n}-\int_{\partial T_{i}} \frac{1}{R_{i}}\left(u^{\varepsilon}-v^{\varepsilon}\right) \nabla w_{i} \cdot \vec{n}\right\} .
\end{aligned}
$$

Let us rewrite the first two summands as

$$
\begin{aligned}
& -\int_{\Omega} w^{\varepsilon} \nabla z^{\varepsilon} \cdot \nabla\left(u^{\varepsilon}-v^{\varepsilon}\right)+\int_{\Omega} \nabla z^{\varepsilon} \cdot \nabla w^{\varepsilon}\left(u^{\varepsilon}-v^{\varepsilon}\right) \\
& =-2 \int_{\Omega} w^{\varepsilon} \nabla z^{\varepsilon} \cdot \nabla\left(u^{\varepsilon}-v^{\varepsilon}\right)-\int_{\Omega} w^{\varepsilon} \Delta z^{\varepsilon}\left(u^{\varepsilon}-v^{\varepsilon}\right) \\
& =\int_{\Omega} \Delta z^{\varepsilon}\left(u^{\varepsilon}-v^{\varepsilon}\right)+2 \int_{\Omega}\left(1-w^{\varepsilon}\right) \nabla z^{\varepsilon} \cdot \nabla\left(u^{\varepsilon}-v^{\varepsilon}\right)+\int_{\Omega}\left(1-w^{\varepsilon}\right) \Delta z^{\varepsilon}\left(u^{\varepsilon}-v^{\varepsilon}\right) .
\end{aligned}
$$

We proceed as follows:

- Step 1: We are going to show for the last two summands in (55) that 


$$
\begin{aligned}
& \left|2 \int_{\Omega}\left(1-w^{\varepsilon}\right) \nabla z^{\varepsilon} \cdot \nabla\left(u^{\varepsilon}-v^{\varepsilon}\right)+\int_{\Omega}\left(1-w^{\varepsilon}\right) \Delta z^{\varepsilon}\left(u^{\varepsilon}-v^{\varepsilon}\right)\right| \\
& \quad \leqslant C(T) \varepsilon^{2}\left\|\nabla\left(u^{\varepsilon}-v^{\varepsilon}\right)\right\|_{L^{2}(\Omega)} .
\end{aligned}
$$

- Step 2: The second step is more tedious. We are going to show that

$$
\begin{aligned}
& \left|\int_{\Omega} \Delta z^{\varepsilon}\left(u^{\varepsilon}-v^{\varepsilon}\right)-\sum_{i}\left\{\int_{\partial T_{i}} z^{\varepsilon}\left(u^{\varepsilon}-v^{\varepsilon}\right) \nabla w_{i} \cdot \vec{n}-\int_{\partial T_{i}} \frac{1}{R_{i}}\left(u^{\varepsilon}-v^{\varepsilon}\right) \nabla w_{i} \cdot \vec{n}\right\}\right| \\
& \quad \leqslant C(T) \varepsilon\left\|\nabla\left(u^{\varepsilon}-v^{\varepsilon}\right)\right\|_{L^{2}(\Omega)} .
\end{aligned}
$$

In the upcoming estimates we will often use the uniform bound (14), which we will not mention explicitly anymore.

Let us start to prove Step 1. By Sobolev's embedding theorem and the Poincaré inequality (40) we find

$$
\left\|u^{\varepsilon}-v^{\varepsilon}\right\|_{L^{6}(\Omega)} \leqslant C(T)\left\|\nabla\left(u^{\varepsilon}-v^{\varepsilon}\right)\right\|_{L^{2}(\Omega)} .
$$

Since $\Delta z^{\varepsilon} \in L^{\infty}\left((0, T) ; L^{3}(\Omega)\right)$ by Corollary 3.2 we obtain with (48) and Hölder's inequality

$$
\int_{\Omega}\left(1-w^{\varepsilon}\right) \Delta z^{\varepsilon}\left(u^{\varepsilon}-v^{\varepsilon}\right) \leqslant C(T) \varepsilon^{2}\left\|\nabla\left(u^{\varepsilon}-v^{\varepsilon}\right)\right\|_{L^{2}(\Omega)} .
$$

Using (27) and again (48) we have

$$
\begin{aligned}
2 \int_{\Omega}\left(1-w^{\varepsilon}\right) \nabla z^{\varepsilon} \cdot \nabla\left(u^{\varepsilon}-v^{\varepsilon}\right) & \leqslant C\left\|1-w^{\varepsilon}\right\|_{L^{5 / 2}(\Omega)}\left\|\nabla z^{\varepsilon}\right\|_{L^{10}(\Omega)}\left\|\nabla\left(u^{\varepsilon}-v^{\varepsilon}\right)\right\|_{L^{2}(\Omega)} \\
& \leqslant C(T) \varepsilon^{2}\left\|\nabla\left(u^{\varepsilon}-v^{\varepsilon}\right)\right\|_{L^{2}(\Omega)}
\end{aligned}
$$

which finishes the proof of Step 1.

Now we proceed to prove Step 2. We compute

$$
\begin{aligned}
& \int_{\Omega} \Delta z^{\varepsilon}\left(u^{\varepsilon}-v^{\varepsilon}\right)-\sum_{i} \int_{\partial T_{i}} z^{\varepsilon}\left(u^{\varepsilon}-v^{\varepsilon}\right) \nabla w_{i} \cdot \vec{n}+\sum_{i} \int_{\partial T_{i}} \frac{1}{R_{i}}\left(u^{\varepsilon}-v^{\varepsilon}\right) \nabla w_{i} \cdot \vec{n} \\
& \stackrel{(24)}{=} \sum_{i}\left\{4 \pi R_{i} \int_{Q_{i}} z^{\varepsilon}\left(u^{\varepsilon}-v^{\varepsilon}\right)-\int_{\partial T_{i}} z^{\varepsilon}\left(u^{\varepsilon}-v^{\varepsilon}\right) \nabla w_{i} \cdot \vec{n}\right\} \\
& \quad+\sum_{i}\left\{\int_{\partial T_{i}} \frac{1}{R_{i}}\left(u^{\varepsilon}-v^{\varepsilon}\right) \nabla w_{i} \cdot \vec{n}-4 \pi \int_{Q_{i}}\left(u^{\varepsilon}-v^{\varepsilon}\right)\right\} \\
& =: I_{1}+I_{2} .
\end{aligned}
$$

The terms $I_{1}$ and $I_{2}$ will both be estimated in a similar fashion. We start with $I_{1}$.

Using the abbreviation $y^{\varepsilon}:=z^{\varepsilon}\left(u^{\varepsilon}-v^{\varepsilon}\right)$ we have

$$
I_{1}=\sum_{i} 4 \pi R_{i} \int_{Q_{i}} y^{\varepsilon}-\int_{Q_{i}} \frac{1}{\varepsilon^{3}}\left[\int_{\partial T_{i}} \nabla w_{i} \cdot \vec{n}\right] y^{\varepsilon}
$$




$$
\begin{aligned}
& +\sum_{i} \int_{Q_{i}} \frac{1}{\varepsilon^{3}}\left[\int_{\partial T_{i}} \nabla w_{i} \cdot \vec{n}\right] y^{\varepsilon}-\int_{\partial T_{i}} y^{\varepsilon} \nabla w_{i} \cdot \vec{n} \\
=: & I_{1 a}+I_{1 b} .
\end{aligned}
$$

We are going to show that

$$
\left|I_{1 a}\right| \leqslant C(T) \varepsilon^{2}\left\|\nabla\left(u^{\varepsilon}-v^{\varepsilon}\right)\right\|_{L^{2}(\Omega)}
$$

which is easy. The main part will be to show that

$$
\left|I_{1 b}\right| \leqslant C(T) \varepsilon\left\|\nabla\left(u^{\varepsilon}-v^{\varepsilon}\right)\right\|_{L^{2}(\Omega)} .
$$

To prove (60) we use (52) and find

$$
\begin{aligned}
& \left|I_{1 a}\right| \leqslant \sum_{i} 4 \pi R_{i}\left|1-\frac{1}{1-2 \varepsilon^{2} R_{i}}\right| \int_{Q_{i}}\left|y^{\varepsilon}\right| \\
& \stackrel{(53)}{\leqslant} C \varepsilon^{2} \sum_{i} R_{i}^{2} \int_{Q_{i}}\left|y^{\varepsilon}\right| \leqslant C \varepsilon^{2} \sum_{i} R_{i}^{2} \varepsilon^{3 / 2}\left(\int_{Q_{i}}\left|y^{\varepsilon}\right|^{2}\right)^{1 / 2} \\
& \stackrel{(39)}{\leqslant} C\left\|z^{\varepsilon}\right\|_{L^{\infty}(\Omega)} \varepsilon^{2} \sum_{i} R_{i}^{3 / 2} \varepsilon^{3 / 2}\left\|\nabla\left(u^{\varepsilon}-v^{\varepsilon}\right)\right\|_{L^{2}\left(Q_{i}\right)} \\
& \stackrel{(27)}{\leqslant} C(T) \varepsilon^{2} \sum_{i} R_{i}^{3 / 2} \varepsilon^{3 / 2}\left\|\nabla\left(u^{\varepsilon}-v^{\varepsilon}\right)\right\|_{L^{2}\left(Q_{i}\right)} \\
& \stackrel{(14)}{\leqslant} C(T) \varepsilon^{2}\left\|\nabla\left(u^{\varepsilon}-v^{\varepsilon}\right)\right\|_{L^{2}(\Omega)} .
\end{aligned}
$$

Now we prove (61). For that we define the function $q^{\varepsilon}$ in $Q_{i}$ as a solution of

$$
\begin{aligned}
-\Delta q^{\varepsilon} & =\frac{4 \pi R_{i}}{1-2 \varepsilon^{2} R_{i}} & & \text { in }\left(Q_{i} \backslash \overline{T_{i}}\right) \cup T_{i}, \\
{\left[\nabla q^{\varepsilon} \cdot \vec{n}\right] } & =-\nabla w_{i} \cdot \vec{n} & & \text { on } \partial T_{i}, \\
\nabla q^{\varepsilon} \cdot \vec{n} & =0 & & \text { on } \partial Q_{i}, \\
\int_{Q_{i}} q^{\varepsilon} & =0 . & &
\end{aligned}
$$

By (52) this problem is well-posed and there exists a solution $q^{\varepsilon} \in H^{1}(\Omega)$ with $\int_{Q_{i}} q^{\varepsilon}=$ 0 and it holds

$$
\int_{Q_{i}} \nabla q^{\varepsilon} \cdot \nabla y^{\varepsilon}=\int_{Q_{i}}\left[\frac{1}{\varepsilon^{3}} \int_{\partial T_{i}} \nabla w_{i} \cdot \vec{n}\right] y^{\varepsilon}-\int_{\partial T_{i}} y^{\varepsilon} \nabla w_{i} \cdot \vec{n} .
$$

Furthermore, since $\int_{Q_{i}} q^{\varepsilon}=0$ and $\Delta q^{\varepsilon}=$ const. in $Q_{i}$, 


$$
\begin{aligned}
\int_{Q_{i}}\left|\nabla q^{\varepsilon}\right|^{2} & =\int_{\partial T_{i}} q^{\varepsilon}\left[\nabla q^{\varepsilon} \cdot \vec{n}\right] \\
& \stackrel{(51)}{\leqslant} \frac{\varepsilon R_{i}}{1-2 \varepsilon^{2} R_{i}} \int_{\partial T_{i}}\left|q^{\varepsilon}\right| \\
& \stackrel{(46)}{\leqslant} C \frac{\varepsilon R_{i}}{1-2 \varepsilon^{2} R_{i}} \varepsilon^{3 / 2}\left(\int_{Q_{i}}\left|\nabla q^{\varepsilon}\right|^{2}\right)^{1 / 2} \\
& \stackrel{(53)}{\leqslant} C \varepsilon^{5 / 2} R_{i}\left(\int_{Q_{i}}\left|\nabla q^{\varepsilon}\right|^{2}\right)^{1 / 2}
\end{aligned}
$$

which gives

$$
\left\|\nabla q^{\varepsilon}\right\|_{L^{2}\left(Q_{i}\right)} \leqslant C R_{i} \varepsilon^{5 / 2} .
$$

Using this estimate in $I_{1 b}$ we obtain

$$
\begin{aligned}
\left|I_{1 b}\right| & \stackrel{(63)}{\leqslant} \sum_{i} \int_{Q_{i}}\left|\nabla q^{\varepsilon} \cdot \nabla y^{\varepsilon}\right| \leqslant \sum_{i}\left\|\nabla q^{\varepsilon}\right\|_{L^{2}\left(Q_{i}\right)}\left\|\nabla y^{\varepsilon}\right\|_{L^{2}\left(Q_{i}\right)} \\
& \stackrel{(64)}{\leqslant} C \varepsilon \sum_{i} R_{i} \varepsilon^{3 / 2}\left\|\nabla y^{\varepsilon}\right\|_{L^{2}\left(Q_{i}\right)} \\
& \leqslant C \varepsilon \sum_{i} R_{i} \varepsilon^{3 / 2}\left(\left\|z^{\varepsilon}\right\|_{L^{\infty}(\Omega)}\left\|\nabla\left(u^{\varepsilon}-v^{\varepsilon}\right)\right\|_{L^{2}\left(Q_{i}\right)}+\left\|\nabla z^{\varepsilon}\right\|_{L^{3}\left(Q_{i}\right)}\left\|u^{\varepsilon}-v^{\varepsilon}\right\|_{L^{6}\left(Q_{i}\right)}\right) \\
& \leqslant C \varepsilon\left\|z^{\varepsilon}\right\|_{L^{\infty}(\Omega)}\left\|\nabla\left(u^{\varepsilon}-v^{\varepsilon}\right)\right\|_{L^{2}(\Omega)}+C \varepsilon\left\|\nabla z^{\varepsilon}\right\|_{L^{3}(\Omega)}\left\|u^{\varepsilon}-v^{\varepsilon}\right\|_{L^{6}(\Omega)} \\
& \stackrel{(27),(40)}{\leqslant} C \varepsilon\left\|\nabla\left(u^{\varepsilon}-v^{\varepsilon}\right)\right\|_{L^{2}(\Omega)}
\end{aligned}
$$

which finishes the proof of (61).

Now we consider term $I_{2}$ in (59) which we write as follows.

$$
\begin{aligned}
I_{2}= & \sum_{i}\left\{\int_{Q_{i}}\left[\frac{1}{\varepsilon^{3}} \int_{\partial T_{i}} \frac{1}{R_{i}} \nabla w_{i} \cdot \vec{n}\right]\left(u^{\varepsilon}-v^{\varepsilon}\right)-4 \pi \int_{Q_{i}}\left(u^{\varepsilon}-v^{\varepsilon}\right)\right\} \\
& +\sum_{i}\left\{\int_{\partial T_{i}} \frac{1}{R_{i}}\left(u^{\varepsilon}-v^{\varepsilon}\right) \nabla w_{i} \cdot \vec{n}-\int_{Q_{i}}\left[\frac{1}{\varepsilon^{3}} \int_{\partial T_{i}} \frac{1}{R_{i}} \nabla w_{i} \cdot \vec{n}\right]\left(u^{\varepsilon}-v^{\varepsilon}\right)\right\} \\
= & : I_{2 a}+I_{2 b} .
\end{aligned}
$$

Analogously to the proof of (60) and (61) one shows

$$
\begin{aligned}
\left|I_{2 a}\right| & \leqslant C \varepsilon^{2} \sum_{i} R_{i} \int_{Q_{i}}\left|u^{\varepsilon}-v^{\varepsilon}\right| \\
& \stackrel{(39)}{\leqslant} C \varepsilon^{2} \sum_{i} R_{i}^{1 / 2} \varepsilon^{3 / 2}\left\|\nabla\left(u^{\varepsilon}-v^{\varepsilon}\right)\right\|_{L^{2}\left(Q_{i}\right)} \\
& \stackrel{(14)}{\leqslant} C \varepsilon^{2}\left\|\nabla\left(u^{\varepsilon}-v^{\varepsilon}\right)\right\|_{L^{2}(\Omega)}
\end{aligned}
$$


and, using $\left(u^{\varepsilon}-v^{\varepsilon}\right) / R_{i}$ as test function in (62),

$$
\begin{aligned}
\left|I_{2 b}\right| & \leqslant \sum_{i} \frac{1}{R_{i}} \int_{Q_{i}}\left|\nabla q^{\varepsilon} \cdot \nabla\left(u^{\varepsilon}-v^{\varepsilon}\right)\right| \\
& \leqslant \sum_{i} \frac{1}{R_{i}}\left\|\nabla q^{\varepsilon}\right\|_{L^{2}\left(Q_{i}\right)}\left\|\nabla\left(u^{\varepsilon}-v^{\varepsilon}\right)\right\|_{L^{2}\left(Q_{i}\right)} \\
& \stackrel{(64)}{\leqslant} C \varepsilon \sum_{i} \varepsilon^{3 / 2}\left\|\nabla\left(u^{\varepsilon}-v^{\varepsilon}\right)\right\|_{L^{2}\left(Q_{i}\right)} \\
& \leqslant C \varepsilon\left\|\nabla\left(u^{\varepsilon}-v^{\varepsilon}\right)\right\|_{L^{2}(\Omega)} .
\end{aligned}
$$

This finishes the proof of Step 2 and thus concludes the proof of Theorem 4.1.

COROLLARY 4.2. - For almost all $t \in(0, \infty)$ it holds

$$
\left\|u^{\varepsilon}(t, \cdot)-\left(u w^{\varepsilon}+g^{\varepsilon}\right)(t, \cdot)\right\|_{H^{1}(\Omega)} \rightarrow 0 .
$$

Proof. - We know from Lemma 3.3 that for almost all $t \in(0, \infty)$

$$
z^{\varepsilon}(t, \cdot) \rightarrow u(t, \cdot) \quad \text { in } W^{1, p}(\Omega) \text { for all } p<\infty .
$$

For these $t$ we have

$$
\begin{aligned}
\left\|\nabla\left(u^{\varepsilon}(t, \cdot)-\left(u w^{\varepsilon}+g^{\varepsilon}\right)(t, \cdot)\right)\right\|_{L^{2}(\Omega)} \leqslant & \left\|\nabla\left(u^{\varepsilon}-v^{\varepsilon}\right)(t, \cdot)\right\|_{L^{2}(\Omega)} \\
& +\left\|\nabla\left[\left(z^{\varepsilon}-u\right) w^{\varepsilon}(t, \cdot)\right]\right\|_{L^{2}(\Omega)} \\
\stackrel{(38)}{\leqslant} & C_{t} \varepsilon+\left\|w^{\varepsilon}(t, \cdot)\right\|_{L^{\infty}(\Omega)}\left\|\nabla\left(z^{\varepsilon}-u\right)(t, \cdot)\right\|_{L^{2}(\Omega)} \\
& +\left\|\nabla w^{\varepsilon}(t, \cdot)\right\|_{L^{2}(\Omega)}\left\|\left(z^{\varepsilon}-u\right)(t, \cdot)\right\|_{L^{\infty}(\Omega)} .
\end{aligned}
$$

Since $\left|w^{\varepsilon}\right| \leqslant 1$ and $\nabla w^{\varepsilon}$ is bounded in $L^{2}(\Omega)$ according to (50) we find with (28) and (40) the convergence as claimed.

\section{Asymptotic expansion for the radii evolution}

The corrector result proved in the previous section allows us to give an error estimate for an asymptotic expansion of $R_{i}$ in terms of $z^{\varepsilon}$.

THEOREM 5.1. - Let $\left\{R_{i}\right\}_{i}$ be the solution to (11) and $z^{\varepsilon}$ the solution to (24). For any $T<\infty$ there exists a constant $C=C(T)$ such that

$$
\underset{t \in(0, T)}{\operatorname{ess} \sup } \sum_{i} \varepsilon^{3}\left|\dot{R}_{i} R_{i}^{2}-\left(R_{i} z^{\varepsilon}\left(X_{i}\right)-1\right)\right| \leqslant C \varepsilon
$$

and

$$
\left|\dot{R}_{i} R_{i}^{2}-\left(R_{i} z^{\varepsilon}\left(X_{i}\right)-1\right)\right| \leqslant C R_{i} \varepsilon^{1 / 2}
$$

holds for almost all $t \in(0, T)$ as long as $R_{i}(t)>0$. 
To prove this result we need a local estimate for the difference $u^{\varepsilon}-z^{\varepsilon}$. With the following lemma we shall give a uniform error estimate of $u^{\varepsilon}-z^{\varepsilon}$ away from the particles $B_{i}$. For that we denote the domain where we cut out the balls of radius $\frac{1}{4} \varepsilon$ by

$$
\widehat{\Omega}^{\varepsilon}:=\Omega \backslash \bigcup_{i: R_{i}>0} B\left(X_{i}, \frac{1}{4} \varepsilon\right)
$$

and a slightly smaller domain by

$$
\widetilde{\Omega}^{\varepsilon}:=\Omega \backslash \bigcup_{i: R_{i}>0} B\left(X_{i}, \frac{3}{8} \varepsilon\right) .
$$

LEMmA 5.2. - For any $T>0$ there exists a constant $C=C(T)$ such that

$$
\left\|\left(u^{\varepsilon}-z^{\varepsilon}\right)(t, \cdot)\right\|_{L^{\infty}\left(\widetilde{\Omega}^{\varepsilon}\right)} \leqslant C \varepsilon^{1 / 2} .
$$

Proof. - To prove this estimate we need three preliminary steps.

- Step 1:

$$
\int_{\widehat{\Omega}^{\varepsilon}}\left|u^{\varepsilon}-z^{\varepsilon}\right|^{2} \leqslant C \varepsilon^{2}
$$

- Step 2:

$$
\int_{\widehat{\Omega}^{\varepsilon}}\left|\nabla\left(u^{\varepsilon}-z^{\varepsilon}\right)\right|^{2} \leqslant C \varepsilon^{2} .
$$

- Step 3:

$$
\int_{\widetilde{\Omega}^{\varepsilon}}\left|D^{2}\left(u^{\varepsilon}-z^{\varepsilon}\right)\right|^{2} \leqslant C .
$$

To prove the first step we write

$$
\int_{\widehat{\Omega}^{\varepsilon}}\left|u^{\varepsilon}-z^{\varepsilon}\right|^{2} \leqslant 2 \int_{\widehat{\Omega}^{\varepsilon}}\left|u^{\varepsilon}-v^{\varepsilon}\right|^{2}+2 \int_{\widehat{\Omega}^{\varepsilon}}\left|z^{\varepsilon}-v^{\varepsilon}\right|^{2} .
$$

By (40) and Theorem 4.1 we get

$$
\int_{\widehat{\Omega}^{\varepsilon}}\left|u^{\varepsilon}-v^{\varepsilon}\right|^{2} \leqslant C \int_{\Omega}\left|\nabla\left(u^{\varepsilon}-v^{\varepsilon}\right)\right|^{2} \leqslant C \varepsilon^{2} .
$$

Moreover we have

$$
\int_{\widehat{\Omega}^{\varepsilon}}\left|z^{\varepsilon}-v^{\varepsilon}\right|^{2}=\sum_{i} \int_{Q_{i \backslash B\left(X_{i}, \frac{1}{4} \varepsilon\right)}}\left|\left(1-w^{\varepsilon}\right) z^{\varepsilon}+g^{\varepsilon}\right|^{2}
$$




$$
\begin{aligned}
& \leqslant\left(\left\|z^{\varepsilon}\right\|_{L^{\infty}(\Omega)}+1\right) \sum_{i}\left(1+\frac{1}{R_{i}^{2}}\right) \int_{Q_{i}}\left|1-w_{i}\right|^{2} \\
& \stackrel{(47)}{\leqslant} C\left(\left\|z^{\varepsilon}\right\|_{L^{\infty}(\Omega)}+1\right) \sum_{i} \varepsilon^{7}\left(1+R_{i}^{2}\right) \\
& \stackrel{(27)}{\leqslant} C \varepsilon^{4}
\end{aligned}
$$

which, together with the previous estimate, concludes the proof of Step 1.

Let us prove Step 2. Using Theorem 4.1 we find

$$
\begin{aligned}
\int_{\widehat{\Omega}^{\varepsilon}}\left|\nabla\left(u^{\varepsilon}-z^{\varepsilon}\right)\right|^{2} & \leqslant 2 \int_{\widehat{\Omega}^{\varepsilon}}\left|\nabla\left(u^{\varepsilon}-v^{\varepsilon}\right)\right|^{2}+2 \int_{\widehat{\Omega}^{\varepsilon}}\left|\nabla\left(v^{\varepsilon}-z^{\varepsilon}\right)\right|^{2} \\
& \stackrel{(38)}{\leqslant} C \varepsilon^{2}+2 \iint_{\widehat{\Omega}^{\varepsilon}}\left|\nabla\left(z^{\varepsilon}\left(1-w^{\varepsilon}\right)+g^{\varepsilon}\right)\right|^{2} \\
\leqslant & C \varepsilon^{2}+4\left[\int_{\widehat{\Omega}^{\varepsilon}}\left|\nabla z^{\varepsilon}\right|^{2}\left|1-w^{\varepsilon}\right|^{2}+\int_{\widehat{\Omega}^{\varepsilon}}\left|\nabla w^{\varepsilon}\right|^{2}\left|z^{\varepsilon}\right|^{2}+\int\left|\nabla g^{\varepsilon}\right|^{2}\right] \\
\leqslant & C \varepsilon^{2}+4\left\|1-w^{\varepsilon}\right\|_{L^{5 / 2}(\Omega)}^{2}|| \nabla z^{\varepsilon} \|_{L^{10}(\Omega)} \\
& +4\left\|z^{\varepsilon}\right\|_{L^{\infty}(\Omega)}^{2} \int_{\widehat{\Omega}^{\varepsilon}}\left|\nabla w^{\varepsilon}\right|^{2}+4 \int_{\widehat{\Omega}^{\varepsilon}}\left|\nabla g^{\varepsilon}\right|^{2} .
\end{aligned}
$$

Using the explicit form of the functions $w_{i}$ we compute

$$
\int_{\widehat{\Omega}^{\varepsilon}}\left|\nabla w^{\varepsilon}\right|^{2} \leqslant 4 \pi \sum_{i} \varepsilon^{6} R_{i}^{2} \int_{\varepsilon / 4}^{\varepsilon / 2} \frac{1}{r^{2}} \mathrm{~d} r \leqslant C \sum_{i} \varepsilon^{5} R_{i}^{2} \leqslant C \varepsilon^{2}
$$

and similarly

$$
\int_{\widehat{\Omega}^{\varepsilon}}\left|\nabla g^{\varepsilon}\right|^{2}=\sum_{i} \int_{T_{i} \backslash B\left(X_{i}, \frac{\varepsilon}{4}\right)} \frac{1}{R_{i}^{2}}\left|\nabla w_{i}\right|^{2} \leqslant C \sum_{i} \varepsilon^{5} \leqslant C \varepsilon^{2} .
$$

Together with (27) and (48) we obtain (69).

To prove Step 3 recall that in $\widehat{\Omega}^{\varepsilon}$

$$
-\Delta\left(u^{\varepsilon}-z^{\varepsilon}\right)=4 \pi\left(R^{\varepsilon} z^{\varepsilon}-\chi^{\varepsilon}\right)=: f^{\varepsilon} \in L^{3}(\Omega) .
$$

Differentiating the equation we have for $j \in\{1,2,3\}$ that

$$
-\Delta \partial_{j}\left(u^{\varepsilon}-z^{\varepsilon}\right)=: \partial_{j} f^{\varepsilon} \in H^{-1}(\Omega) .
$$

Let $\eta \in C_{0}^{\infty}\left(\widehat{\Omega}^{\varepsilon}\right)$ be a cut off function such that $\eta \equiv 1$ in $\widetilde{\Omega}^{\varepsilon},\|\nabla \eta\|_{L^{\infty}} \leqslant C / \varepsilon$ and $\left\|D^{2} \eta\right\|_{L^{\infty}} \leqslant C / \varepsilon^{2}$. Taking $\partial_{j}\left(u^{\varepsilon}-z^{\varepsilon}\right) \eta^{2}$ as a test function in (71) we get 


$$
\begin{aligned}
\int_{\widehat{\Omega}^{\varepsilon}} \eta^{2}\left|\nabla \partial_{j}\left(u^{\varepsilon}-z^{\varepsilon}\right)\right|^{2}= & \left\langle\partial_{j} f^{\varepsilon}, \eta^{2} \partial_{j}\left(u^{\varepsilon}-z^{\varepsilon}\right)\right\rangle \\
& \quad-2 \int_{\widehat{\Omega}^{\varepsilon}} \nabla \partial_{j}\left(u^{\varepsilon}-z^{\varepsilon}\right) \cdot \nabla \eta \partial_{j}\left(u^{\varepsilon}-z^{\varepsilon}\right) \eta \\
= & : I+I I .
\end{aligned}
$$

With Young's inequality and (69) we obtain

$$
\begin{aligned}
|I I| & \leqslant \frac{1}{4} \int_{\widehat{\Omega}^{\varepsilon}} \eta^{2}\left|\nabla \partial_{j}\left(u^{\varepsilon}-z^{\varepsilon}\right)\right|^{2}+C \int_{\widehat{\Omega}^{\varepsilon}}|\nabla \eta|^{2}\left|\nabla\left(u^{\varepsilon}-z^{\varepsilon}\right)\right|^{2} \\
& \leqslant \frac{1}{4} \int_{\widehat{\Omega}^{\varepsilon}} \eta^{2}\left|\nabla \partial_{j}\left(u^{\varepsilon}-z^{\varepsilon}\right)\right|^{2}+C
\end{aligned}
$$

Furthermore

$$
\begin{aligned}
|I| \leqslant & \int_{\widehat{\Omega}^{\varepsilon}}\left|f^{\varepsilon} \partial_{j}\left(\eta^{2} \partial_{j}\left(u^{\varepsilon}-z^{\varepsilon}\right)\right)\right| \\
\leqslant & 2 \int_{\widehat{\Omega}^{\varepsilon}}\left|\partial_{j}\left(u^{\varepsilon}-z^{\varepsilon}\right)\right|\left|f^{\varepsilon}\right||\eta||\nabla \eta|+\int_{\widehat{\Omega}^{\varepsilon}}\left|\nabla \partial_{j}\left(u^{\varepsilon}-z^{\varepsilon}\right)\right|\left|f^{\varepsilon}\right| \eta^{2} \\
\leqslant & \int_{\widehat{\Omega}^{\varepsilon}}\left|f^{\varepsilon}\right|^{2} \eta^{2}+\int_{\widehat{\Omega}^{\varepsilon}}\left|\nabla\left(u^{\varepsilon}-z^{\varepsilon}\right)\right|^{2}|\nabla \eta|^{2} \\
& +4 \int\left|f^{\varepsilon}\right|^{2} \eta^{2}+\frac{1}{4} \int_{\widehat{\Omega}^{\varepsilon}} \eta^{2}\left|\nabla \partial_{j}\left(u^{\varepsilon}-z^{\varepsilon}\right)\right|^{2} \\
\stackrel{(69)}{\leqslant} & \frac{1}{4} \int_{\widehat{\Omega}^{\varepsilon}} \eta^{2}\left|\nabla \partial_{j}\left(u^{\varepsilon}-z^{\varepsilon}\right)\right|^{2}+C .
\end{aligned}
$$

Thus we find in (72)

$$
\int_{\Omega^{\varepsilon}}\left|\nabla \partial_{j}\left(u^{\varepsilon}-z^{\varepsilon}\right)\right|^{2} \leqslant \int_{\widehat{\Omega}^{\varepsilon}} \eta^{2}\left|\nabla \partial_{j}\left(u^{\varepsilon}-z^{\varepsilon}\right)\right|^{2} \leqslant C
$$

which concludes the proof of Step 3. The conclusion will follow by an interpolation argument. By Steps 1 and 2 we obtain with Sobolev's embedding theorem that

$$
\left\|u^{\varepsilon}-z^{\varepsilon}\right\|_{L^{6}\left(\widetilde{\Omega}^{\varepsilon}\right)} \leqslant C \varepsilon .
$$

The Gagliardo-Nirenberg inequality [15] gives

$$
\left\|u^{\varepsilon}-z^{\varepsilon}\right\|_{L^{\infty}\left(\widetilde{\Omega}^{\varepsilon}\right)} \leqslant C\left\|D^{2}\left(u^{\varepsilon}-z^{\varepsilon}\right)\right\|_{L^{2}\left(\widetilde{\Omega}^{\varepsilon}\right)}^{1 / 2}\left\|u^{\varepsilon}-z^{\varepsilon}\right\|_{L^{6}\left(\widetilde{\Omega}^{\varepsilon}\right)}^{1 / 2}+C\left\|u^{\varepsilon}-z^{\varepsilon}\right\|_{L^{6}\left(\widetilde{\Omega}^{\varepsilon}\right)} .
$$

With (70) and (75) we find (67). 
We are now in the position to prove Theorem 5.1.

Proof of Theorem 5.1. - Recall that $w_{i}=0$ on $\partial B_{i}$. Hence

$$
\begin{aligned}
\dot{R}_{i} R_{i}^{2} \stackrel{(11)}{=} & \frac{1}{4 \pi \varepsilon^{3}} \int_{\partial B_{i}} \nabla u^{\varepsilon} \cdot \vec{n} \\
= & \frac{1}{4 \pi \varepsilon^{3}} \int_{\partial B_{i}} \nabla v^{\varepsilon} \cdot \vec{n}+\frac{1}{4 \pi \varepsilon^{3}} \int_{\partial B_{i}}\left(1-w_{i}\right) \nabla\left(u^{\varepsilon}-v^{\varepsilon}\right) \cdot \vec{n} \\
\stackrel{(35)}{=} & \frac{1}{4 \pi \varepsilon^{3}} \int_{\partial B_{i}}\left(z^{\varepsilon}-\frac{1}{R_{i}}\right) \nabla w_{i} \cdot \vec{n}+\frac{1}{4 \pi \varepsilon^{3}} \int_{T_{i} \backslash \overline{B_{i}}} \nabla\left(u^{\varepsilon}-v^{\varepsilon}\right) \cdot \nabla w_{i} \\
& +\frac{1}{4 \pi \varepsilon^{3}} \int_{T_{i} \backslash \overline{B_{i}}}\left(1-w_{i}\right) \Delta v^{\varepsilon}
\end{aligned}
$$

and we find

$$
\begin{aligned}
\dot{R}_{i} R_{i}^{2}- & \left(R_{i} z^{\varepsilon}\left(X_{i}\right)-1\right) \\
= & \left(\frac{1}{4 \pi \varepsilon^{3}} \int_{\partial B_{i}} z^{\varepsilon} \nabla w_{i} \cdot \vec{n}-z^{\varepsilon}\left(X_{i}\right) R_{i}\right)+\left(1-\frac{1}{4 \pi \varepsilon^{3}} \int_{\partial B_{i}} \frac{1}{R_{i}} \nabla w_{i} \cdot \vec{n}\right) \\
& +\frac{1}{4 \pi \varepsilon^{3}} \int_{T_{i} \backslash \overline{B_{i}}}\left(1-w_{i}\right) \Delta v^{\varepsilon}+\frac{1}{4 \pi \varepsilon^{3}} \int_{T_{i} \backslash \overline{B_{i}}} \nabla\left(u^{\varepsilon}-v^{\varepsilon}\right) \cdot \nabla w_{i} .
\end{aligned}
$$

Since

$$
\nabla w_{i} \cdot \vec{n}=\frac{1}{\varepsilon^{3} R_{i}} \frac{1}{1-2 \varepsilon^{2} R_{i}} \quad \text { on } \partial B_{i}
$$

and $z^{\varepsilon} \in C^{0,1 / 2}(\Omega)$ we find with (53) that

$$
\begin{aligned}
\left|\frac{1}{4 \pi \varepsilon^{3}} \int_{\partial B_{i}} z^{\varepsilon} \nabla w_{i} \cdot \vec{n}-z^{\varepsilon}\left(X_{i}\right) R_{i}\right| & \leqslant 4 \varepsilon^{2} R_{i}\left\|z^{\varepsilon}\right\|_{L^{\infty}(\Omega)}+R_{i}\left|f_{\partial B_{i}} z^{\varepsilon}-z^{\varepsilon}\left(X_{i}\right)\right| \\
& \leqslant 4 \varepsilon^{2} R_{i}\left\|z^{\varepsilon}\right\|_{L^{\infty}(\Omega)}+R_{i}\left(\varepsilon^{3} R_{i}\right)^{1 / 2}\left\|z^{\varepsilon}\right\|_{C^{0,1 / 2}} \\
& \stackrel{(16)}{ } \leqslant\left(\left\|z^{\varepsilon}\right\|_{C^{0,1 / 2}}\right) \varepsilon R_{i} .
\end{aligned}
$$

Again with (53) we have

$$
\left|1-\frac{1}{4 \pi \varepsilon^{3}} \int_{\partial B_{i}} \frac{1}{R_{i}} \nabla w_{i} \cdot \vec{n}\right| \leqslant 4 \varepsilon^{2} R_{i}
$$

Furthermore, with (24), (35), (47), (49) and the fact that $0 \leqslant w_{i} \leqslant 1$ we have

$$
\frac{1}{4 \pi \varepsilon^{3}} \int_{T_{i} \backslash \overline{B_{i}}}\left(1-w_{i}\right) \Delta v^{\varepsilon}=\frac{1}{4 \pi \varepsilon^{3}} \int_{T_{i} \backslash \overline{B_{i}}}\left(1-w_{i}\right)\left(\Delta z^{\varepsilon} w_{i}+2 \nabla z^{\varepsilon} \cdot \nabla w_{i}\right)
$$




$$
\begin{aligned}
&= \frac{1}{4 \pi \varepsilon^{3}} \int_{T_{i} \backslash \overline{B_{i}}}\left(1-w_{i}\right)\left(\left(R_{i} z^{\varepsilon}-1\right)+2 \nabla z^{\varepsilon} \cdot \nabla w_{i}\right) \\
& \leqslant \frac{1}{4 \pi \varepsilon^{3}}\left(\left(R_{i}\left\|z^{\varepsilon}\right\|_{L^{\infty}(\Omega)}+1\right)\left\|1-w_{i}\right\|_{L^{1}\left(Q_{i}\right)}\right. \\
&\left.+\left\|1-w_{i}\right\|_{L^{12 / 5}\left(Q_{i}\right)}\left\|\nabla z^{\varepsilon}\right\|_{L^{12}(\Omega)}\left\|\nabla w_{i}\right\|_{L^{2}\left(Q_{i}\right)}\right) \\
& \leqslant C\left(\left\|z^{\varepsilon}\right\|_{W^{2,3}(\Omega)}+1\right)\left(R_{i} \varepsilon^{2}+R_{i}\left(R_{i} \varepsilon^{7 / 2}\right)^{1 / 2}\right) \\
& \stackrel{(16)}{\leqslant} C\left(\left\|z^{\varepsilon}\right\|_{W^{2,3}(\Omega)}+1\right) R_{i} \varepsilon^{5 / 4} .
\end{aligned}
$$

We use (78), (79) and (80) in (77) and obtain

$$
\left|\dot{R}_{i} R_{i}^{2}-\left(R_{i} z^{\varepsilon}\left(X_{i}\right)-1\right)\right| \leqslant C\left(\left\|z^{\varepsilon}\right\|_{W^{2,3}(\Omega)}+1\right) R_{i} \varepsilon+\frac{1}{4 \pi \varepsilon^{3}}\left|\int_{T_{i}} \nabla\left(u^{\varepsilon}-v^{\varepsilon}\right) \cdot \nabla w_{i}\right|
$$

To prove (65) we multiply the last equation with $\varepsilon^{3}$, sum over $i$ and use Corollary 3.2, (38) and (50) to find

$$
\begin{aligned}
\sum_{i} \varepsilon^{3}\left|\dot{R}_{i} R_{i}^{2}-\left(R_{i} z^{\varepsilon}\left(X_{i}\right)-1\right)\right| & \leqslant C\left(\varepsilon+\left\|\nabla w^{\varepsilon}\right\|_{L^{2}(\Omega)}\left\|\nabla\left(u^{\varepsilon}-v^{\varepsilon}\right)\right\|_{L^{2}(\Omega)}\right) \\
& \leqslant C \varepsilon .
\end{aligned}
$$

Finally, integrating by parts and taking into account that $v^{\varepsilon}=z^{\varepsilon}$ on $\partial T_{i}$ we obtain with (51) that

$$
\begin{aligned}
\frac{1}{4 \pi \varepsilon^{3}} \int_{T_{i} \backslash \overline{B_{i}}} \nabla\left(u^{\varepsilon}-v^{\varepsilon}\right) \cdot \nabla w_{i} & =\frac{1}{4 \pi \varepsilon^{3}} \int_{\partial T_{i}}\left(u^{\varepsilon}-z^{\varepsilon}\right) \nabla w_{i} \cdot \vec{n} \\
& \leqslant\left\|u^{\varepsilon}-z^{\varepsilon}\right\|_{L^{\infty}\left(\partial T_{i}\right)} C R_{i} \\
& \stackrel{(67)}{\leqslant} C R_{i} \varepsilon^{1 / 2}
\end{aligned}
$$

which together with (81) finishes the proof of Theorem 5.1.

\section{Acknowledgements}

We gratefully acknowledge support by the European Community through the TMR program Nonlinear parabolic partial differential equations: methods and applications, ERB FMRX CT98 0201.

\section{REFERENCES}

[1] Alikakos N., Fusco G., The equations of Ostwald ripening for dilute systems, J. Stat. Phys. 95 (5/6) (1999) 851-866.

[2] Alikakos N., Fusco G., Ostwald ripening for dilute systems under quasistationary dynamics, Comm. Math. Phys., to appear. 
[3] Chen X., Global asymptotic limit of solutions of the Cahn-Hilliard equation, J. Diff. Geometry 44 (1996) 262-311.

[4] Chen X., Hong J., Yi F., Existence, uniqueness and regularity of classical solutions of the Mullins-Sekerka problem, Comm. Part. Diff. Eq. 21 (1996) 1705-1727.

[5] Cioranescu D., Murat F., A strange term coming from nowhere, in: Cherkaev A., Kohn R.V. (Eds.), Topics in the Mathematical Modelling of Composite Materials, Birkhäuser, 1997, pp. 45-94.

[6] Escher J., Simonett G., Classical solutions for the quasi-stationary Stefan problem with surface tension, in: Demuth M. (Ed.), Papers associated with the International Conference on Partial Differential Equations, Potsdam, Germany, June 29-July 2, 1996, AkademieVerlag, 1996, pp. 98-104.

[7] Grisvard P., Elliptic Problems in Nonsmoooth Domains, Pitman, Boston, 1985.

[8] Kacimi H., Murat F., Estimation de l'erreur dans des problemes de Dirichlet ou apparait un terme etrange, in: Partial Differential Equations and the Calculus of Variations. Essays in Honor of Ennio De Giorgi, Birkhäuser, 1989, pp. 661-696.

[9] Langer J.S., An introduction to the kinetics of first order phase transitions, in: Godrẽche C. (Ed.), Solids Far from Equilibrium, Cambridge University Press, 1992, pp. 297-362.

[10] Lifshitz I.M., Slyozov V.V., The kinetics of precipitation from supersaturated solid solutions, J. Phys. Chem. Solids 19 (1961) 35-50.

[11] Niethammer B., Derivation of the LSW-theory for Ostwald ripening by homogenization methods, Arch. Rat. Mech. Anal. 147 (2) (1999) 119-178.

[12] Niethammer B., Otto F., Ostwald Ripening: The screening length revisited, Calc. Var. and PDE 13 (1) (2001) 33-68.

[13] Niethammer B., Pego R.L., Non-self-similar behavior in the LSW theory of Ostwald ripening, J. Stat. Phys. 95 (5/6) (1999) 867-902.

[14] Niethammer B., Pego R.L., On the initial-value problem in the Lifshitz-Slyozov-Wagner theory of Ostwald ripening, SIAM J. Math. Anal. 31 (3) (2000) 457-485.

[15] Nirenberg L., An extended interpolation inequality, Ann. Scuola Norm. Sup. Pisa Ser. III 20 (1966) 733-737.

[16] Velázquez J.J.L., On the effect of stochastic fluctuations in the dynamics of the LifshitzSlyozov-Wagner model, J. Stat. Phys. (2000) 57-113.

[17] Voorhees P.W., The theory of Ostwald ripening, J. Stat. Phys. 38 (1985) 231-252.

[18] Wagner C., Theorie der Alterung von Niederschlägen durch Umlösen, Z. Elektrochemie 65 (1961) 581-594.

[19] Ziemer W.P., Weakly Differentiable Functions, Springer, New York, 1989. 